\title{
Act-and-Wait Concept for Continuous-Time Control Systems With Feedback Delay
}

\author{
Tamás Insperger
}

\begin{abstract}
The act-and-wait control concept is introduced for continuous-time control systems with feedback delay associated with infinite poles. The point of the method is that the feedback is periodically switched on (act) and off (wait) during the control. It is shown that if the duration of waiting (when the control is switched off) is larger than the feedback delay, then the system can be represented by a finite dimensional monodromy matrix, and a finite number of eigenvalues describe stability. This way, the infinite dimensional pole placement problem is reduced to a finite dimensional one. The efficiency of the method is demonstrated on a case study.
\end{abstract}

Index Terms-Delay effects, Floquet theory, optimal control, periodic control, pole assignment, stability.

\section{INTRODUCTION}

O

YNSIDER the linear system

$$
\dot{\mathbf{x}}(t)=\mathbf{A x}(t)+\mathbf{B u}(t)
$$

where $\mathbf{x}(t) \in \mathbb{R}^{n}$ is the state vector, $\mathbf{u}(t) \in \mathbb{R}^{m}$ is the input, and $\mathbf{A} \in \mathbb{R}^{n \times n}$ and $\mathbf{B} \in \mathbb{R}^{n \times m}$ are given constant matrices. Consider the autonomous delayed feedback controller

$$
\mathbf{u}(t)=\mathbf{D} \mathbf{x}(t-\tau)
$$

where $\mathbf{D} \in \mathbb{R}^{m \times n}$ is a constant matrix and $\tau$ is the delay of the feedback. We assume that the delay is a fixed parameter of the control system and cannot be eliminated or tuned during the control design. There are several sources of such time delays, e.g., acquisition of response and excitation data, information transmission, online data processing, computation, and application of control forces.

System (1), with controller (2), implies the delay-differential equation (DDE)

$$
\dot{\mathbf{x}}(t)=\mathbf{A} \mathbf{x}(t)+\mathbf{B D} \mathbf{x}(t-\tau) .
$$

Due to the time delay, this system has an infinite number of poles (also called characteristic roots or characteristic exponents) determined by the transcendental characteristic equation

$$
\operatorname{det}\left(\lambda \mathbf{I}-\mathbf{A}-\mathbf{B D e}^{-\tau \lambda}\right)=0 .
$$

Manuscript received November 21, 2005. Manuscript received in final form March 31, 2006. Recommended by Associate Editor Z. Wang. This work was supported in part by the Hungarian Academy of Sciences under the János Bolyai Research Scholarship and by the Hungarian National Science Foundation under Grants OTKA F047318 and OTKA T043368.

The author is with the Department of Applied Mechanics, Budapest University of Technology and Economics, H-1521 Budapest, Hungary (e-mail: inspi@mm.bme.hu).

Digital Object Identifier 10.1109/TCST.2006.876938
The system is asymptotically stable if all the poles are located in the left half of the complex plane. Stability conditions for the systems's parameters can be given by monitoring the number of unstable poles (see, e.g., [1]-[3]).

We introduce the decay ratio $\rho=\mathrm{e}^{\operatorname{Re} \lambda_{1}}$, where $\lambda_{1}$ is the relevant (rightmost) pole, i.e., $\operatorname{Re} \lambda_{1} \geq \operatorname{Re} \lambda_{i}, i=2,3, \ldots, \infty$. This ratio is a measure of the average error decay over a unit period, since $|\mathbf{x}(t+1)| \leq \rho|\mathbf{x}(t)|$. Optimization of (1) and (2) raises the following pole placement problem. For the given matrices $\mathbf{A}$ and $\mathbf{B}$ and for given feedback delay $\tau$, we are looking for matrix D, so that $\operatorname{Re} \lambda_{1}$ and, consequently, $\rho$ are as small as possible. The difficulty of this problem is that an infinite amount of poles should be controlled by a finite number of control parameters, i.e., by the elements of matrix $\mathbf{D}$.

An effective way of managing the pole placement problem is through the use of periodic controllers. Several papers have been published on the stabilization effect of periodic feedback for both discrete-time [5], [4], and continuous-time systems [6]-[8]. An efficient technique is the use of generalized sampled-data hold functions by sampling the output periodically and using special hold functions [9]. Pulse-step control [10] and controlsignal shaping [11] should also be mentioned here as control concepts with step-wise varying input.

In this brief, a special case of periodic controllers, the act-andwait controller, is introduced for continuous-time systems with delayed feedback. The point of the method is that the control term is periodically switched off and on during the control so that the duration of being switched off is larger than the feedback delay. It is shown that by using this method, the system can be described by an $n \times n$ monodromy matrix. The stability is then determined by $n$ poles (by the eigenvalues of the monodromy matrix) as opposed to the infinite number of roots of (4). This way, the dimension of the pole placement problem is reduced to $n$.

In the second half of the brief, a case study is presented: a second-order system with delayed feedback. It is shown that the application of the act-and-wait method radically simplifies pole placing difficulties, improves stability properties, and results in a deadbeat control even in the case when the system can not be stabilized with the original autonomous controller.

The act-and-wait method was already introduced in [12] for semi-discrete systems with piecewise constant delayed term associated with a finite dimensional spectrum. In the present brief, the method is adopted for continuous-time delayed systems associated with infinite dimensional spectrum.

\section{ACT-AND-WAIT CONTROLLER}

We introduce the act-and-wait controller

$$
\mathbf{u}(t)=g(t) \mathbf{D} \mathbf{y}(t-\tau)
$$


where $g(t)$ is the $T$-periodic act-and-wait switching function defined as

$$
g(t)= \begin{cases}0, & \text { if } 0 \leq t<t_{w} \\ 1, & \text { if } t_{w} \leq t<t_{w}+t_{a}=T .\end{cases}
$$

Using controller (5) instead of (2), the delayed feedback term is switched off for a period of length $t_{w}$ (wait), and it is switched on for a period of length $t_{a}$ (act). This is a special case of periodic controllers.

System (1), with controller (5), can be written in the timeperiodic DDE form

$$
\dot{\mathbf{x}}(t)=\mathbf{A} \mathbf{x}(t)+g(t) \mathbf{B D} \mathbf{x}(t-\tau) .
$$

According to the Floquet theory of DDEs, stability is determined by the nonzero eigenvalues of the system's monodromy operator, called also characteristic multipliers (see, e.g., [13]). The system is asymptotically stable if all the characteristic multipliers are inside the unit circle of the complex plane.

For the time-periodic system (7), the decay ratio can be defined as $\rho=\left|\mu_{1}\right|^{1 / T}$, where $\mu_{1}$ is the relevant characteristic multiplier, i.e., $\left|\mu_{1}\right| \geq\left|\mu_{i}\right|, i=2,3, \ldots, \infty$. This decay ratio can be used to compare act-and-wait control systems with different periods $T$, to the autonomous system (3).

Now, the optimization problem can be composed in the following way. For given matrices $\mathbf{A}$ and $\mathbf{B}$ and for given feedback delay $\tau$, we are looking for matrix $\mathbf{D}$, and parameters $t_{w}$ and $t_{a}$ so that $\left|\mu_{1}\right|$ and, consequently, $\rho$ are as small as possible.

In the case $t_{w}<\tau,(7)$ has infinite number of characteristic multipliers, therefore, pole placing is still rather complicated, similarly to the autonomous case (3). However, if $t_{w} \geq \tau$, then the monodromy operator of (7) becomes finite dimensional and can be represented as an $n \times n$ matrix as it is shown below.

First, assume that $t_{w} \geq \tau$ and $0<t_{a} \leq \tau$. In this case, (7) can be considered as an ordinary differential equation (ODE) in $\left[0, t_{w}\right)$ and as aDE in $\left[t_{w}, T\right)$. If $t \in\left[0, t_{w}\right)$, then $g(t)=0$ (the delayed term is switched off), and the solution of (7), associated with the initial state $\mathbf{x}(0)$, can be given as

$$
\mathbf{x}(t)=\mathrm{e}^{\mathbf{A} t} \mathbf{x}_{0} \quad t \in\left[0, t_{w}\right)
$$

If $t \in\left[t_{w}, T\right.$ ), then $g(t)=1$ (the delayed term is switched on). Since $t_{a} \leq \tau$, and the solution over the interval $\left[0, t_{w}\right)$ is already given by (8), system (7) can be written in the form

$$
\dot{\mathbf{x}}(t)=\mathbf{A x}(t)+\mathbf{B D} \mathrm{e}^{\mathbf{A}(t-\tau)} \mathbf{x}(0) \quad t \in\left[t_{w}, T\right) .
$$

Solving (9) as an ODE over $\left[t_{w}, T\right)$ with $\mathbf{x}\left(t_{w}\right)=\mathrm{e}^{\mathbf{A} t_{w}} \mathbf{x}(0)$ as an initial condition, we obtain

$$
\mathbf{x}(T)=\underbrace{\left(\mathrm{e}^{\mathbf{A} T}+\int_{t_{w}}^{T} \mathrm{e}^{\mathbf{A}(T-s)} \mathbf{B D} \mathrm{e}^{\mathbf{A}(s-\tau)} \mathrm{d} s\right)}_{\mathbf{\Phi}} \mathbf{x}(0) .
$$

This way, we constructed an $n \times n$-discrete map over the period $T$ for the initial state $\mathbf{x}(0)$ using the piecewise solutions of (7). This means that the monodromy operator of (7) has $n$ nonzero eigenvalues that are equal to the eigenvalues of the transition matrix $\boldsymbol{\Phi}$. All the other (infinitely many) eigenvalues of the monodromy operator are zero. Consequently, system (7) has $n$ characteristic multipliers that are equal to the eigenvalues of matrix $\boldsymbol{\Phi}$ that is actually the monodromy matrix of the system.

Consider now the case when the period of acting can not be smaller than the time delay due to any reasons (e.g., physical limitations of the controller), that is, $t_{w} \geq \tau$ and $t_{a}>\tau$. In this case, the monodromy matrix can be determined by stepwise integration over subsequent intervals. First, the solution over $\left[0, t_{w}\right)$ should be determined similarly to $(8)$. Then, the solutions can be determined over the intervals $\left[t_{w}, t_{w}+\tau\right),\left[t_{w}+\right.$ $\left.\tau, t_{w}+2 \tau\right)$, etc., by step-by-step substituting the solution of the previous interval into the delayed term. If $k \tau<t_{a} \leq(k+1) \tau$, then $\mathbf{x}(T)$ is obtained after $k+2$ succeeding integration.

For example, if $\tau<t_{a} \leq 2 \tau$, then piecewise integration over $\left[0, t_{w}\right),\left[t_{w}, t_{w}+\tau\right)$, and $\left[t_{w}+\tau, T\right)$ yields the $n \times n$ monodromy matrix

$$
\begin{aligned}
\mathbf{\Phi}= & \mathrm{e}^{\mathbf{A} T}+\int_{t_{w}}^{T} \mathrm{e}^{\mathbf{A}(T-s)} \mathbf{B D} \mathrm{e}^{\mathbf{A}(s-\tau)} \mathrm{d} s \\
& +\int_{\boldsymbol{t}_{w}+\tau}^{T} \mathrm{e}^{\mathbf{A}\left(T-s_{1}\right)} \mathbf{B D} \int_{t_{w}}^{s_{1}-\tau} \mathrm{e}^{\mathbf{A}\left(s_{1}-s_{2}-\tau\right)} \mathbf{B D} \\
& \times \mathrm{e}^{\mathbf{A}\left(s_{2}-\tau\right)} \mathrm{d} s_{2} \mathrm{~d} s_{1} .
\end{aligned}
$$

The main conclusion, regarding the general system (7), is that if $t_{w} \geq \tau$ and $t_{a}>0$, then an $n \times n$ monodromy matrix can always be constructed using the piecewise solutions of (7). Consequently, $n$ eigenvalues determine the stability properties of the system as opposed to the infinite number of poles of the system with the original autonomous controller (2). This way, the infinite dimensional-pole placement problem is reduced to an $n$-dimensional one: $n$ eigenvalues of $\boldsymbol{\Phi}$ should be placed using the control parameters in $\mathbf{D}$.

Note that the above statement does not imply that arbitrary pole placement of the resulted monodromy matrix is always possible. Actually, the placement of the eigenvalues of $\boldsymbol{\Phi}$ is still a rather complex problem, since they depend nonlinearly on the components of $\mathbf{D}$. Therefore, the stabilization problem of the general system (7) is not investigated here. Only a case study is presented in the next section about the pole optimization of a second-order system with delayed feedback.

\section{CASe Study}

Consider the second-order system with delayed feedback described by in (1), (5), and (6) with

$$
\begin{aligned}
& \mathbf{A}=\left[\begin{array}{cc}
0 & 1 \\
-a & 0
\end{array}\right] \quad \mathbf{B}=\left[\begin{array}{l}
0 \\
1
\end{array}\right] \\
& \mathbf{D}=\left[\begin{array}{l}
-d_{1} \\
-d_{2}
\end{array}\right]^{T} \quad \tau=1 .
\end{aligned}
$$

If $g(t) \equiv 1\left(t_{w}=0\right)$, then the characteristic equation of the corresponding autonomous system reads

$$
\lambda^{2}+a+d_{1} \mathrm{e}^{-\lambda}+d_{2} \lambda \mathrm{e}^{-\lambda}=0 .
$$

This system has an infinite number of poles that can not arbitrarily be placed using the two control parameters $d_{1}$ and $d_{2}$. Moreover, if $a<-2$, then the autonomous system cannot even 
(a)

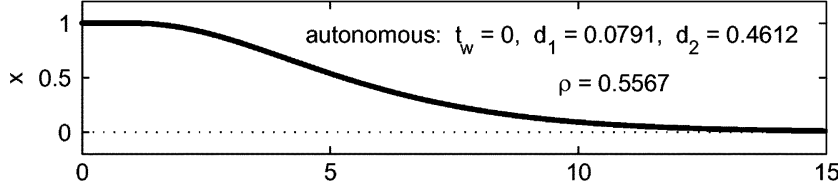

(b)

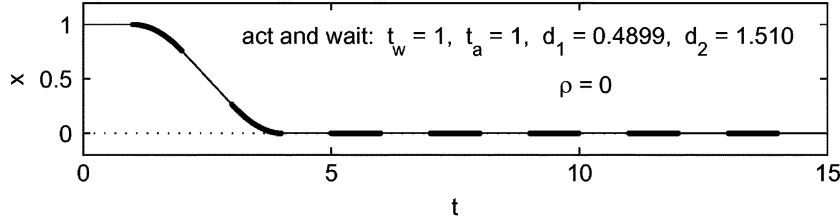

Fig. 1. Time histories for (12) with $a=0$ using (a) autonomous and (b) actand-wait controller.

be stabilized, i.e., the system is unstable for all $\left(d_{1}, d_{2}\right)$ pairs (see, e.g., [1]).

In the following subsections, cases $a=0$ and $a=-4$ will be investigated. Optimal control parameters corresponding to the smallest possible decay ratios will be determined for the autonomous control case $\left(t_{w}=0\right)$ and for the act-and-wait case with $t_{w}=1$ and $t_{a}=1$.

\section{A. Case $a=0$}

This case describes a feedback stabilized double integrator with input delay. The system can be stabilized using an autonomous controller with $t_{w}=0$, and pole optimization gives the optimal control parameters $d_{1}=0.0791, d_{2}=0.4612$ with the relevant pole $\lambda_{1}=-0.5858$ (see, e.g., [14]). The corresponding decay ratio is $\rho=\left|\mathrm{e}^{\lambda_{1}}\right|=0.5567$.

If the act-and-wait control concept is used with $t_{w}=1=\tau$ and $t_{a}=1=\tau$, then the system can be represented by the $2 \times 2$ monodromy matrix

$$
\boldsymbol{\Phi}=\left[\begin{array}{cc}
1-\frac{1}{2} d_{1} & 2-\frac{1}{6} d_{1}-\frac{1}{2} d_{2} \\
-d_{1} & 1-\frac{1}{2} d_{1}-d_{2}
\end{array}\right]
$$

given by (10). The pole placement problem is now reduced to the placement of the two eigenvalues of $\boldsymbol{\Phi}$ using the control parameters $d_{1}, d_{2}$. It can be seen that $d_{1}$ and $d_{2}$ can be chosen so that both eigenvalues are zero, that is, $\rho=0$ ! These optimal parameters are $d_{1}=-12+2 \sqrt{39}=0.4899$ and $d_{2}=14-$ $2 \sqrt{39}=1.510$. If these parameters are used then $\Phi^{2}=\mathbf{0}$, that is, the system is deadbeat in $2 T$ period!

Time histories for the autonomous and the act-and-wait cases are shown in Fig. 1. The simulation was performed using the semidiscretization technique [15]. Thick lines denote the periods of acting and thin lines denote waiting. In the autonomous case in Fig. 1(a), the control is continuously active, and the convergence is relatively slow. In Fig. 1(b), the time history corresponding to the act-and-wait control shows that the system ac-

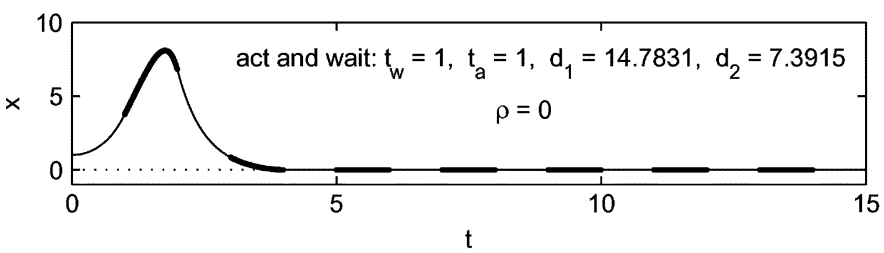

Fig. 2. Time history for (12) with $a=-4$ using act-and-wait controller.

tually converges to zero within period $2 T$. In the period $\left[0, t_{w}\right)$, the delayed term is switched off (we are waiting), in the period $\left[t_{w}, T\right)$, the controller is switched on forcing the system to zero (we are acting). In the next act-and-wait period, the system stops at zero completing the deadbeat convergence.

\section{B. Case $a=-4$}

In this case, matrix $\mathbf{A}$ describes an unstable system (like the inverted pendulum in [1]). This system can not be stabilized using an autonomous controller since $a<-2$.

If the act-and-wait controller is used with $t_{w}=1=\tau$ and $t_{a}=1=\tau$, then the monodromy matrix can be given according to $(15)$, shown at the bottom of the page.

It can be seen that this matrix can be stabilized, moreover, both eigenvalues can be placed to the origin using the control parameters $d_{1}=14.7831$ and $d_{2}=7.3915$, that is, deadbeat control can be achieved! Time history corresponding to the optimal deadbeat case is shown in Fig. 2. It can be seen that $x(t)$ grows exponentially in the first wait period $\left[0, t_{w}\right)$ since $\mathbf{A}$ is unstable, then, during the first act period $\left[t_{w}, T\right)$, the growing tendency of $x(t)$ is reversed, and the deadbeat convergence is completed in the next act-and-wait period.

\section{CONCLUSION}

The act-and-wait control method was introduced for linear $n$-dimensional continuous-time control systems with feedback delay $\tau$. It was shown that the infinite dimensional pole placement problem of the delayed system can be reduced to an $n$-dimensional one, if the feedback is periodically switched off and on with switch-off period $t_{w} \geq \tau$. The point is that the switched system can be described by an $n \times n$ monodromy matrix, consequently, stability properties are described by $n$ eigenvalues.

As a case study, a second-order system with delayed feedback was investigated. It was shown that the number of poles can be reduced from infinity to 2 by applying the act-and-wait method, and deadbeat control can be attained even in the case when the original autonomous controller cannot stabilize the system.

The main conclusion of this paper is that the act-and-wait concept provides an alternative for control systems with feedback delays. The traditional way is the continuous use of small

$$
\boldsymbol{\Phi}=\left[\begin{array}{cc}
27.3082-0.9067 d_{1}-0.9744 d_{2} & 13.6450-0.2436 d_{1}-0.9067 d_{2} \\
54.5798-2.7878 d_{1}-3.62698 d_{2} & 27.3082-0.9067 d_{1}-2.7878 d_{2}
\end{array}\right]
$$


control gains according to Fig. 1(a), when a cautious, slow feedback is applied with small gains resulting in slow convergence. The proposed alternative way is the act-and-wait control concept, when large control gains are used in the acting period and zero gains are used in the waiting period according to Fig. 1(b). Although it might seem unnatural not to actuate during the wait period, the act-and-wait concept is still a natural control logic for time-delayed systems. This is the way, for example, that one would adjust the shower temperature considering the delay between the controller (tap) and the sensed output (skin).

\section{REFERENCES}

[1] G. Stépán, Retarded Dynamical Systems. White Plains, NY: Longman, 1989.

[2] F. M. Atay, "Balancing the inverted pendulum using position feedback," Appl. Math. Lett., vol. 12, no. 5, pp. 51-56, Dec. 1999.

[3] N. Olgac and R. Sipahi, "An exact method for the stability analysis of time-delayed linear time-invariant (LTI) systems," IEEE Trans. Autom. Control, vol. 47, no. 5, pp. 793-977, May 2002.

[4] D. Aeyels and J. L. Willems, "Pole assignment for linear time-invariant systems by periodic memoryless output feedback," Automatica, vol. 28, no. 6, pp. 1159-1168, Nov. 1992.

[5] P. P. Khargonekar, K. Poplla, and A. Tannenbaum, "Robust control of linear time-invariant plants using periodic compensation," IEEE Trans. Autom. Control, vol. AC-30, no. 11, pp. 1088-1096, Nov. 1985.
[6] T. Insperger and G. Stépán, "Stability chart for the delayed Mathieu equation," in Proc. Royal Society Math. Phys. Eng. Sci., 2002, pp. 1989-1998.

[7] W. Michiels, S.-I. Niculescu, and L. Moreau, "Using delays and timevarying gains to improve the static output feedback stabilizability of linear systems: a comparision," IMA J. Math. Contr. Inform., vol. 21, no. 4, pp. 393-418, Dec. 2004.

[8] J. C. Allwright, A. Astolfi, and H. P. Wong, "A note on asymptotic stabilization of linear systems by periodic, piece-wise constant output feedback," Automatica, vol. 41, no. 2, pp. 339-344, Feb. 2005.

[9] P. Kabamba, "Control of linear systems using generalized sampleddata hold functions," IEEE Trans. Autom. Control, vol. 32, no. 9, pp. 772-783, Sep. 1987.

[10] A. Wallén and K. J. Åström, "Pulse-step control," in Proc. 15th IFAC World Congress, 2002, CD ROM.

[11] Q.-C. Zhong and C.-C. Hang, "Control of processes with dead time and input constraints using control signal shaping," Proc. Inst. Elect. Eng. Contr. Theory Appl., vol. 151, no. 4, pp. 473-480, Jul. 2004.

[12] T. Insperger and G. Stépán, "Optimization of digital control with delay by periodic variation of the gain parameters," in Proc. IFAC Workshop Adaptation Learning Contr. Signal Process. IFAC Workshop Periodic Contr. Syst., 2004, pp. 145-150.

[13] M. Farkas, Periodic Motions. New York: Springer-Verlag, 1994.

[14] W. Michiels and D. Roose, "An eigenvalue based approach for the robust stabilization of linear time-delay systems," Int. J. Control, vol. 76, no. 7, pp. 678-686, May 2003.

[15] T. Insperger and G. Stépán, "Semi-discretization method for delayed systems," Int. J. Numer. Methods Eng., vol. 55, no. 5, pp. 503-518, Oct. 2002. 